\title{
Aspirina em Baixa Dosagem em Pacientes Com Diabete Melito: Riscos e Benefícios em Relação às Complicaçōes Macro e Microvasculares
}

\begin{abstract}
RESUMO
O uso de aspirina é recomendado como estratégia de prevenção cardiovascular em pacientes com diabete melito. Em decorrência do risco de eventos hemorrágicos e da hipótese de que poderia haver um agravamento das complicações microvasculares associado ao uso da aspirina, tem havido importante sub-utilização dessa terapia. Entretanto, está definido que o uso de aspirina não piora a retinopatia diabética e existem evidências de que também não afeta a função renal em doses usuais (150 $\mathrm{mg} / \mathrm{dia}$ ). Por outro lado, pacientes com diabete melito parecem necessitar de doses maiores do agente antiplaquetário, o que sugere que esses indivíduos apresentem a chamada "resistência à aspirina". Os mecanismos dessa resistência ainda não estão completamente esclarecidos, estando provavelmente relacionados à atividade plaquetária intrínseca anormal. Portanto, o emprego de terapêuticas antiplaquetárias alternativas ou a administração de doses maiores de aspirina $(150-300 \mathrm{mg} / \mathrm{dia})$ devem ser melhor avaliados em relação a um aumento da eficácia na prevenção da doença cardiovascular e também a possíveis efeitos nas complicações microvasculares no diabete melito. (Arq Bras Endocrinol Metab 2007;51/3:457-465)
\end{abstract}

Descritores: Diabete melito; Aspirina; Nefropatia diabética; Prevenção cardiovascular; Retinopatia diabética

\section{ABSTRACT}

Low-dose Aspirin in Patients with Diabete Melitus: Risks and Benefits Regarding Macro and Microvascular Complications.

Aspirin is recommended as cardiovascular disease prevention in patients with diabetes mellitus. Due to the increased risk of bleeding and because of the hypothesis that there could be a worsening of microvascular complications related to aspirin, there has been observed an important underutilization of the drug. However, it is now known that aspirin is not associated with a deleterious effect on diabetic retinopathy and there is evidence indicating that it also does not affect renal function with usual doses (150 $\mathrm{mg} / \mathrm{d}$ ). On the other hand, higher doses may prove necessary, since recent data suggest that diabetic patients present the so called "aspirin resistance". The mechanisms of this resistance are not yet fully understood, being probably related to an abnormal intrinsic platelet activity. The employment of alternative antiplatelet strategies or the administration of higher aspirin doses (150-300 $\mathrm{mg} / \mathrm{d}$ ) should be better evaluated regarding effective cardiovascular disease prevention in diabetes as well as the possible effects on microvascular complications. (Arq Bras Endocrinol Metab 2007;51/3:457-465)

Keywords: Diabetes mellitus; Aspirin; Diabetic nephropathy; Cardiovascular prevention; Diabetic retinopathy artigo original

\author{
Eduardo G. Camargo \\ JORGE LUIZ GROSS \\ LetícIa S. WeINERT \\ JOEL LAVINSKY \\ SANDRA P. SILVEIRO
}

Serviço de Endocrinologia do Hospital de Clínicas de Porto Alegre, Universidade Federal do Rio Grande do Sul, Porto Alegre, RS.

Recebido em 02/06/06 Revisado em 27/10/06 Aceito em 02/11/06 
$\mathrm{H}$ Á UMA EXPECTATIVA DE aumento da prevalência de diabete melito (DM) nos próximos anos em todo o mundo. No Brasil, estima-se que o número de casos de DM passe de 4,6 milhões em 2000 para 11,3 milhões em 2030 (1). Com o aumento do número de casos, haverá também um grande acréscimo na proporção de complicações micro e macrovasculares associadas à doença.

O DM é considerado um fator de risco equivalente à doença cardiovascular $(\mathrm{DCV})(2,3)$ e sua presença aumenta em duas a quatro vezes a incidência de doença coronariana quando comparada a indivíduos sem DM (4). Além disso, esses indivíduos estão propensos a um aumento de morbidade e mortalidade decorrentes de complicações como a nefropatia (ND), a neuropatia e a retinopatia diabéticas (RD) (5). Nos Estados Unidos, o DM é a principal causa de insuficiência renal terminal e cegueira em adultos (6). No Brasil, cerca de 30\% dos indivíduos com DM desenvolvem microalbuminúria (7) e a ND é responsável por $25 \%$ dos casos em hemodiálise, sendo que $40 \%$ dos indivíduos morrem no primeiro ano de tratamento (8), principalmente devido à DCV (9).

A presença de microalbuminúria aumenta de forma independente o risco de DCV em indivíduos com DM (10). Estudos recentes sobre trombose e aterosclerose têm demonstrado que as complicações microvasculares e a mortalidade cardiovascular parecem compartilhar processos patogênicos comuns, envolvendo disfunção endotelial $(11,12)$, inflamação em baixo grau $(12,13)$ e estresse oxidativo (14).

Entre as estratégias de prevenção da DCV em indivíduos com DM, o uso de aspirina tem sido recomendado de forma enfática (15). Apesar disso, até $25 \%$ dos pacientes com DCV e $65 \%$ daqueles com outros fatores de risco não recebem a aspirina como estratégia para evitar um evento cardiovascular (16). Rolka e cols. (16) descrevem que o risco de hemorragia digestiva, desconhecimento ou dúvida sobre a real eficácia do uso de aspirina em pacientes com DM foram as principais causas para a prescrição indesejavelmente reduzida da medicação. Da mesma forma, o suposto risco de nefropatia associada ao uso de anti-inflamatórios (17) ou o temor da ocorrência de hemorragia retiniana em pacientes com RD também poderiam ser fatores limitantes à prescrição de aspirina.

O objetivo deste artigo é revisar aspectos do uso de aspirina em indivíduos com DM, analisando os riscos e benefícios em relação às complicações macro e microvasculares associadas à doença.

\section{MECANISMO DE AÇĀO DA ASPIRINA}

A enzima ciclooxigenase (Cox) está envolvida na síntese de prostanóides (figura 1), catalisando a conversão do ácido araquidônico em prostaglandina

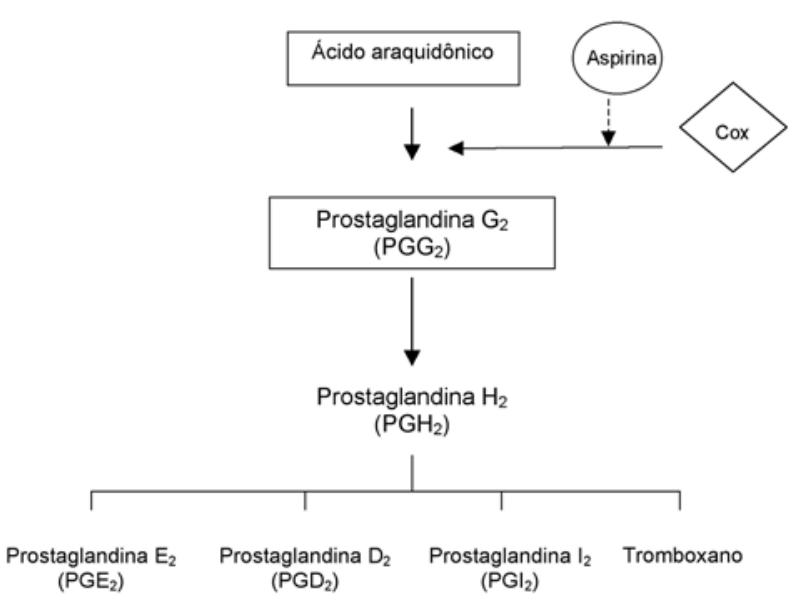

Figura 1. Síntese de prostaglandinas e tromboxano pela via de ciclooxigenase (Cox) e mecanismo de ação da aspirina. (-) inibição.

$\mathrm{G}_{2}\left(\mathrm{PGG}_{2}\right)(18,19)$. Por ação de uma enzima peroxidase, a $\mathrm{PGG}_{2}$ é convertida em prostaglandina $\mathrm{H}_{2}$ $\left(\mathrm{PGH}_{2}\right)$, que é a precursora direta das várias formas de prostaglandinas e tromboxano.

O passo seguinte ocorre nos diferentes tecidos e, dependendo do tipo de célula envolvida, a $\mathrm{PGH}_{2}$ pode originar $\mathrm{PGE}_{2}, \mathrm{PGD}_{2}, \mathrm{PGI}_{2}$ (ou prostaciclina) e tromboxano $\left(\mathrm{TXA}_{2}\right)(20)$. A $\mathrm{PGF}_{2} \downarrow$, sintetizada a partir da $\mathrm{PGE}_{2}$, aumenta a vasoconstrição, a broncoconstrição e a contração da musculatura lisa, enquanto que as prostaglandinas $\mathrm{PGD}_{2}$ e $\mathrm{PGE}_{2}$ induzem a vasodilatação e estimulam os nociceptores. A prostaciclina é produzida pelas células endoteliais e sua função é inibir a agregação plaquetária e causar vasodilatação arterial. $\mathrm{O} \mathrm{TXA}_{2}$ é predominantemente produzido pelas plaquetas e tem importante efeito vasoconstritor e estimulador da agregação plaquetária (19). A prostaciclina e o $\mathrm{TXA}_{2}$ têm, portanto, ações opostas e um importante efeito na regulação do tônus vascular e da agregação plaquetária.

As formas conhecidas da Cox são a Cox-l e a Cox-2 e suas diferentes funções na síntese de prostanóides têm sido descritas (20). A aspirina atua principalmente na Cox-1 e seu efeito é decorrente da acetilação do grupo hidroxila do resíduo de serina na posição 529 da cadeia polipeptídica da Cox, causando uma inibição irreversível desta enzima (21). O resultado é a diminuição da conversão do ácido araquidônico em prostaglandina $\mathrm{G}_{2}$ e, conseqüentemente, redução da síntese de prostaglandinas e $\mathrm{TXA}_{2}$.

$\mathrm{O}$ efeito antitrombótico da aspirina decorre da inibição da Cox-1 em vários tecidos (21). Nas plaquetas, o uso de doses tão baixas quanto $30 \mathrm{mg} /$ dia de aspirina resulta em inibição quase que completa da síntese de $\mathrm{TXA}_{2}(22)$. Isso ocorre antes mesmo que a 
droga atinja a circulação sistêmica, provavelmente pelo efeito de acetilação da Cox-1 plaquetária na circulação portal. Como a inibição da Cox-1 é irreversível, o efeito da aspirina permanece por 8 a 10 dias, que é o tempo de meia-vida plaquetária. Após a interrupção do tratamento com a aspirina, a atividade da Cox recupera-se lentamente, conforme o aparecimento de novas plaquetas na circulação sangüínea. Isto explica o paradoxo de como uma medicação com meia-vida plasmática de aproximadamente 20 minutos pode ter um efeito antitrombótico prolongado mesmo quando administrada somente uma vez ao dia. Da mesma forma, como a inibição da Cox é permanente, a administração de repetidas doses de aspirina tem um efeito cumulativo e, após 7 a 10 dias de uso, observa-se uma supressão completa da síntese de $\mathrm{TXA}_{2}$ plaquetário.

Além do efeito antitrombótico e anti-inflamatório, estudos experimentais têm demonstrado que a aspirina pode ter propriedades anti-oxidantes, reduzindo a produção de superóxido pelas células endoteliais induzida pela angiotensina II e causando relaxamento vascular e diminuição da pressão arterial (23). Da mesma forma, em modelos experimentais, o uso de aspirina reduziu a excreção urinária de albumina e a hipertrofia ventricular induzidas pela angiotensina II, através da inibição do fator pró-inflamatório NF- $\downarrow$ B (24).

\section{ASPIRINA E DOENÇA MACROVASCULAR}

Conforme a recomendação da American Diabetes Association $(A D A)$, o uso de aspirina na dose de 75-162 mg ao dia está indicado além da prevenção secundária, como estratégia de prevenção primária de DCV para pacientes com DM e idade acima de 40 anos ou com pelo menos um fator de risco para DCV (tabela 1) (15) ou, de acordo com a US Task Force (25), quando a avaliação do risco de um primeiro evento coronariano em 10 anos é maior ou igual a 10\% (figura 2). O uso de aspirina não é recomendado a pacientes abaixo de 21 anos pelo risco de Síndrome de

Tabela 1. Indicações para o uso de aspirina como prevenção primária em pacientes com diabete melito.

Idade acima de 40 anos *

Tabagismo

Dislipidemia

Hipertensão arterial sistêmica

Micro ou macroalbuminúria

História familiar de cardiopatia isquêmica

* Pacientes de 30 a 40 anos devem receber aspirina na presença de outros fatores de risco. Não administrar a pacientes abaixo de 21 anos pelo risco de Síndrome de Reye.
Reye, a qual é caracterizada por um quadro viral, associado à encefalopatia metabólica progressiva, com edema cerebral e hipertensão intracraniana (cefaléia, vômitos e irritabilidade, evoluindo para alteração do nível de consciência e coma) e esteatose hepática microvesicular com insuficiência hepática.

\section{Prevenção primária de DCV}

A estratégia de prevenção primária visa a evitar o surgimento de um primeiro evento coronariano e/ou cerebrovascular. Não existem estudos de prevenção primária exclusivamente em pacientes com DM e as recomendações atuais são baseadas nos principais ensaios sobre aspirina e prevenção cardiovascular (tabela 1 ).

O relato inicial do uso de aspirina na prevenção primária de DCV foi feito pelo U.S. Physicians' Health Study (USPHS) em 1989 (26). Neste estudo randomizado, envolvendo 22.071 homens, o uso de aspirina (325 mg em dias alternados) reduziu em 44\% o risco do primeiro infarto agudo do miocárdio (IAM) quando comparado ao placebo. No USPHS, nos 533 indivíduos com DM houve uma redução de $60 \%$ no risco do primeiro IAM quando comparado ao placebo. Em contraste, este benefício não foi evidenciado no British Doctors' Trial (27), um estudo com baixo poder estatístico que comparou o uso de aspirina (500 $\mathrm{mg} / \mathrm{dia})$ com controle aberto em 5.139 homens aparentemente saudáveis.

Entretanto, estudos subseqüentes confirmaram o benefício do uso da aspirina em prevenir o primeiro evento cardiovascular. O Thrombosis Prevention Trial (28) comparou o efeito da aspirina $(75 \mathrm{mg} / \mathrm{dia})$ ao placebo em 5.085 homens com pelo menos dois fatores de risco para DCV e observou uma redução significativa de $32 \%$ no risco do primeiro IAM entre aqueles que utilizaram aspirina. No estudo Hypertension Optimal Treatment (HOT) (29), que incluiu 18.790 pacientes hipertensos $(9.907$ homens e 8.883 mulheres), dos quais cerca de $10 \%$ tinham DM, o uso de aspirina $(75 \mathrm{mg} / \mathrm{dia}$ ) reduziu em $36 \%$ o risco do primeiro IAM e em $15 \%$ o risco de qualquer evento vascular importante. Neste estudo, a análise do subgrupo de mulheres não mostrou uma redução significativa do primeiro IAM.

O estudo Primary Prevention Project (PPP) (30) avaliou o uso de aspirina ( $100 \mathrm{mg} / \mathrm{dia}$ ) em 4.495 indivíduos ( 1.912 homens e 2.583 mulheres) com pelo menos um fator de risco para $\mathrm{DCV}$, entre o quais 1.031 indivíduos tinham DM. Foi observada uma significativa redução de $23 \%$ no risco de todos os eventos cardiovasculares e redução de $44 \%$ de morte 


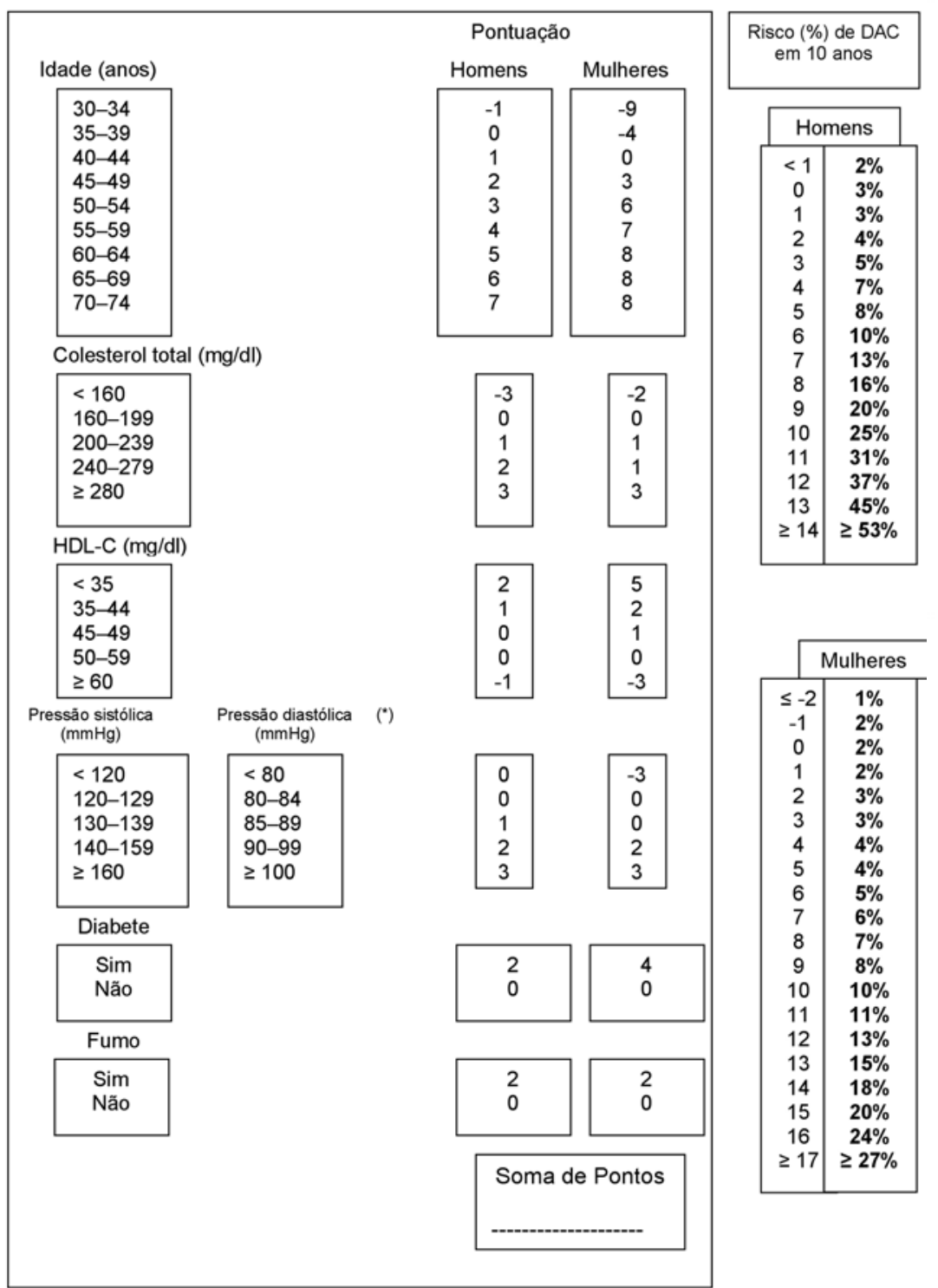

(*) Quando os valores de pressão sistólica e diastólica discordarem, usar o mais alto.

Figura 2. Escore de risco de doença coronariana (DAC) - escore de Framingham.

Tabela 2. Estudos que avaliaram o efeito de diferentes doses de aspirina na prevenção primária de eventos cardiovasculares.

\begin{tabular}{|c|c|c|c|}
\hline Estudo & Ano & Dose & Efeito \\
\hline Physician's Health Study (24) & 1988 & $\begin{array}{c}325 \mathrm{mg} / \mathrm{dias} \\
\text { alternados }\end{array}$ & $\downarrow$ IAM \\
\hline British Doctor's Trial (25) & 1988 & 500 mg/dia & ausente \\
\hline Thrombosis Prevention Trial (26) & 1998 & $75 \mathrm{mg} / \mathrm{dia}$ & $\downarrow I A M$ \\
\hline Hypertension Optimal Treatment (27) & 1998 & $75 \mathrm{mg} / \mathrm{dia}$ & ฟAM \\
\hline Primary Prevention Project (28) & 2001 & $100 \mathrm{mg} / \mathrm{dia}$ & $\downarrow \mathrm{AM}$ \\
\hline Women's Health Study (31) & 2005 & $\begin{array}{l}100 \mathrm{mg} / \mathrm{dias} \\
\text { alternados }\end{array}$ & $\downarrow \mathrm{AVCi}$ \\
\hline
\end{tabular}

IAM: infarto agudo do miocárdio; AVCi: acidente vascular cerebral isquêmico. 
de origem cardiovascular entre aqueles que usavam a aspirina, com a análise combinada para homens e mulheres. No entanto, a análise dos pacientes com DM mostrou uma redução significativa de risco de eventos cardiovasculares de $10 \%$, menor do que aquela dos indivíduos não-diabéticos (31), sugerindo que doses mais baixas de aspirina poderiam ser menos efetivas na prevenção primária nestes indivíduos. Como este estudo apresentava baixo poder estatístico, apenas novas análises poderão definir essa questão.

Uma metanálise (32) envolvendo os resultados dos cinco principais estudos de prevenção primária concluiu que o uso de aspirina reduz significativamente o risco do primeiro IAM em $32 \%$ e de eventos vasculares combinados em $15 \%$. Em relação à prevenção de acidente vascular isquêmico e morte cardiovascular, não houve uma diminuição significativa devido ao reduzido número de casos relatados. Adicionalmente, a análise dos resultados no grupo de mulheres, a partir dos dados dos estudos HOT e PPP, sugere que o uso de aspirina também possa ser benéfico para prevenção do primeiro IAM neste grupo. Em contraste, o Women's Health Study (33) recentemente comparou o uso de aspirina ( $100 \mathrm{mg}$ em dias alternados) e placebo em 39.876 mulheres cuja maioria tinha menos de $5 \%$ de risco de doença coronariana em 10 anos e menos de 3\% tinham DM, por um período de 10 anos. Esse estudo não demonstrou uma redução significativa no risco de um primeiro IAM com o uso de aspirina, apesar de evidenciar uma redução significativa no risco de acidente vascular cerebral (AVC) isquêmico. O efeito benéfico cardiovascular da aspirina foi maior no subgrupo de mulheres com mais de 65 anos (10\% da população do estudo), mas com um aumento na incidência de hemorragia digestiva nesse grupo. De fato, uma metanálise recente mostrou que o uso de aspirina exerce benefício cardiovascular diferente em ambos os sexos, reduzindo significativamente o risco de IAM em homens (32\%) e de AVC isquêmico em mulheres (24\%) (34). Essa metanálise também demostrou que o uso de aspirina nas doses recomendadas está associado a um aumento significativo do risco de sangramento cerebral e, principalmente, digestivo, reforçando a necessidade de avaliar risco e benefício para prescrição do agente anti-plaquetário.

Em conclusão, o uso de aspirina de liberação entérica é formalmente recomendado pela ADA como estratégia de prevenção primária de eventos cardiovasculares em indivíduos com DM (15). Entretanto, estudos envolvendo especificamente pacientes com DM são necessários para estabelecer o real benefício do uso de aspirina neste grupo.

\section{Prevenção secundária}

O uso de aspirina é recomendado como estratégia de prevenção secundária em indivíduos com DM que apresentam história de IAM, angina, revascularização arterial, acidente vascular cerebral isquêmico transitório ou permanente ou doença vascular periférica (15).

A metanálise mais recente conduzida pelo grupo Antithrombotic Trialists' Collaboration (ATC) analisou 287 estudos de prevenção secundária com aspirina em 212.000 pacientes com alto risco para um novo evento cardiovascular (35). A dose de aspirina nos estudos foi de 75 a $325 \mathrm{mg} / \mathrm{dia}$, sendo que as doses mais baixas $(75-150 \mathrm{mg} /$ dia) foram tão efetivas quanto as doses mais elevadas. Nessa metanálise, uma redução significativa de $23 \%$ no risco de novos eventos vasculares foi observada com o uso de aspirina. Nos cerca de 4.900 indivíduos com DM, a terapia com aspirina foi associada a uma redução significativa, mas de apenas 7\% nos eventos vasculares.

Portanto, o uso de aspirina como estratégia de prevenção secundária de DCV é recomendado em indivíduos que já apresentaram um evento cardiovascular, incluindo aqueles com DM. Nesse grupo, o uso de aspirina em doses baixas parece não ser tão eficaz quanto naqueles sem DM, e o uso de doses maiores ou outro agente antitrombótico, como o clopidogrel, tem sido sugerido e deve ser adicionalmente investigado (36). O estudo CHARISMA (Clopidogrel for High Atherothrombotic Risk and Ischemic Stabilization, Management, and Avoidance), publicado no New England Journal of Medicine de abril de 2006, foi um estudo prospectivo, randomizado, duplo-cego e controlado com placebo, que analisou o uso de aspirina (75-162 mg/dia) com placebo em comparação ao uso de aspirina com clopidogrel (75 mg/dia) em 15.603 pacientes ou com DCV evidente ou múltiplos fatores de risco. A conclusão do estudo foi a de que a dupla combinação ofereceria um benefício potencial em pacientes com DCV definida, requerendo, ainda, no entanto, confirmação adicional e também de que não deverá ser empregada em pacientes sem DCV estabelecida (37).

\section{ASPIRINA E DOENÇA MICROVASCULAR}

\section{Aspirina e nefropatia diabética}

A possibilidade do desenvolvimento de nefrite intersticial e o efeito inibitório sobre a produção de prostaglandinas vasodilatadoras renais decorrentes do uso de aspirina poderiam ter um efeito deletério sobre a função renal (17). Por outro lado, a ND tem sido 
associada com inflamação em baixo-grau e níveis elevados de proteína $C$ reativa $(12,13)$ em indivíduos com DM, e o uso de aspirina poderia ter um efeito benéfico sobre a função renal devido à sua ação antiinflamatória. Da mesma forma, a hipótese de que a hiperglicemia poderia causar lesão microvascular através de mecanismos envolvendo estresse oxidativo (14) e a evidência experimental de que a aspirina poderia ter propriedades anti-oxidativas (23) poderiam contribuir para um possível efeito benéfico do medicamento na ND. Adicionalmente, as propriedades antiplaquetárias da aspirina poderiam ter um efeito favorável sobre a excreção urinária de albumina (EUA) $(38,39)$ por reduzir a produção renal de $\mathrm{TXA}_{2}$ e fatores de crescimento produzidos pelas plaquetas e supostamente envolvidos na lesão glomerular de pacientes com DM (38).

Donadio e cols. avaliaram o efeito da aspirina em doses elevadas ( $325 \mathrm{mg} 3$ vezes ao dia) associada ao dipiridamol em 28 pacientes com DM tipo 1 e EUA $>1,0 \mathrm{~g} /$ dia, por um período de 4 anos, e observaram uma redução significativa da albuminúria em $25 \%$ dos pacientes (38). Esses achados vieram a sugerir que o tratamento com dipiridamol e aspirina poderia estabilizar a função renal por reduzir a hipersensibilidade plaquetária e a produção de $\mathrm{TXA}_{2}$ pelas plaquetas e/ou tecido renal.

Khajehdehi e cols. também avaliaram o uso de aspirina ( $1000 \mathrm{mg} /$ dia $)$ associada ou não ao dipiridamol em pacientes com DM tipo 2 e EUA $\geq 500 \mathrm{mg} / \mathrm{dia}$, comparado com placebo, e observaram uma redução significativa de $15 \%, 14 \%$ e $37 \%$ na EUA nos grupos usando aspirina, dipiridamol ou a associação de ambos, respectivamente (40).

Por outro lado, aspirina em doses menores, na faixa recomendada para prevenção da DCV, não tem mostrado interferência na função renal de indivíduos com DM. O uso de aspirina (150 mg/dia) por 4 semanas em 16 pacientes com DM tipo l (4l) e 31 pacientes com DM tipo 2 (42) com EUA $>30 \mathrm{mg} / 24$ horas não resultou em modificação da função renal (EUA e taxa de filtração glomerular) desses pacientes. Da mesma forma, o uso de aspirina em dose mais elevada $(300 \mathrm{mg} / \mathrm{dia})$ por 8 semanas em 18 pacientes com DM 2 microalbuminúricos também não modificou a EUA e a taxa de filtração glomerular (TFG), quando comparado com placebo (43).

Como o paciente com DM e especialmente com ND deve usar simultaneamente aspirina e inibidores da enzima conversora da angiotensina (IECA), tem relevância a questão da interação entre esses medicamentos. Análises a partir de ensaios clínicos de tratamento de doença coronariana ou insuficiência cardíaca sugerem que o uso associado de aspirina poderia atenuar os benefícios cardiovasculares dos IECA mediados pelo aumento da produção de bradicinina (ou vice-versa) $(44,45)$. Uma metanálise recente, incluindo seis estudos e 22.060 pacientes, analisou o benefício cardiovascular do uso de IECA na presença e na ausência de aspirina (46). Foi observado, de forma significativa, um efeito menor da terapia com IECA na prevenção de IAM na presença de aspirina quando comparado com a ausência do agente antiplaquetário. Por outro lado, houve uma tendência, embora não significativa, na redução das admissões hospitalares por insuficiência cardíaca no grupo usando a associação. Não houve uma diferença significativa entre os grupos em relação à mortalidade total, incidência de acidente vascular cerebral ou insuficiência cardíaca. Em relação à possível interação da aspirina sobre a ação antiproteinúrica dos IECA, não foi observada perda desse efeito em estudo recente em pacientes DM2 microalbuminúricos (43).

A interação entre aspirina e os bloqueadores dos receptores da angiotensina II (BRA), medicamentos que atuam na via bradicinina-prostaglandina, também foi avaliada (47). A hipótese é de que os receptores AT 2 da angiotensina, estimulados durante o bloqueio dos receptores AT 1 , estariam envolvidos em uma produção compensatória de bradicinina. A análise de um subgrupo de pacientes do estudo Losartan Intervention for Endpoint Reduction in Hypertension (LIFE) mostrou uma maior redução de eventos cardiovasculares no grupo utilizando losartan associado à aspirina quando comparado com aqueles que não usaram o agente antiplaquetário, sugerindo, portanto, uma interação benéfica entre as medicações (47).

Em conclusão, o uso de aspirina em doses baixas está indicado em pacientes com DM e ND como estratégia de prevenção primária de DCV e, até o presente momento, não foram observados efeitos deletérios sobre a função renal. Por outro lado, o efeito antiproteinúrico da aspirina deve ser melhor estudado, assim como sua interação com os IECA e BRA.

\section{Aspirina e retinopatia diabética}

$\mathrm{O}$ efeito da aspirina sobre a $\mathrm{RD}$ foi definido pelo clássico Early Treatment Diabetic Retinopathy Study (ETDRS) (48). Este foi um estudo prospectivo que avaliou o efeito da aspirina $(650 \mathrm{mg} /$ dia $)$ em cerca de 3.700 pacientes com DM, cuja hipótese inicial era de que a medicação poderia retardar a progressão da $\mathrm{RD}$, já que havia relatos de que as alterações plaquetárias e o aumento da síntese de $\mathrm{TXA}_{2}$ em pacientes com DM 
poderiam resultar em estreitamento dos capilares da retina. Os resultados do estudo não mostraram um efeito da aspirina em retardar a progressão da $\mathrm{RD}$, mas também não houve um aumento no risco de hemorragia vítrea relacionado à medicação quando comparado com o placebo. A presença de RD, assim como de ND, está associada a um aumento da mortalidade cardiovascular, que é proporcionalmente maior conforme a gravidade da retinopatia no DM tipo 2 e a perda visual no DM tipo 1 (49).

Em conclusão, o uso de aspirina não oferece risco de hemorragia vítrea ou piora a progressão da doença retiniana em pacientes com RD.

\section{CONSIDERAÇÕES ESPECIAIS}

\section{DM e "resistência à aspirina"}

O fenômeno de "resistência à aspirina" tem sido amplamente discutido $(50,51)$ e inclui os conceitos de resistência laboratorial e resistência clínica ao uso do agente anti-plaquetário. A resistência laboratorial é definida como a incapacidade da aspirina de inibir a produção plaquetária de $\mathrm{TXA}_{2}$ ou inibir a agregação plaquetária dependente do $\mathrm{TXA}_{2}$, medidas através de testes específicos (50). A resistência clínica é definida como a incapacidade da aspirina em prevenir eventos cardiovasculares isquêmicos apesar do uso de doses terapêuticas recomendadas.

Uma revisão recente (51), entretanto, sugere que, na prática clínica, o uso da expressão "falência terapêutica" seria mais apropriado do que "resistência" à aspirina, já que vários fatores poderiam estar envolvidos em uma menor resposta terapêutica do agente anti-plaquetário, tais como aderência inadequada ao tratamento (52), hipertensão arterial (53), hipercolesterolemia (54) e uso de anti-inflamatórios (21). Assim como em pacientes com cardiopatia isquêmica e acidente vascular cerebral $(53,55)$, evidências recentes têm sugerido que o uso de doses usuais de aspirina em pacientes com DM parece ter um benefício cardiovascular menor quando comparado a indivíduos sem $\mathrm{DM}(32,36)$. A atividade plaquetária intrínseca anormal parece contribuir diretamente na diminuição da ação da aspirina em pacientes com DM (56-58). O controle glicêmico inadequado contribui para diminuição de sensibilidade das plaquetas à aspirina devido à competição entre a reação de acetilação induzida pela aspirina e a reação de glicação de proteínas resultante da hiperglicemia crônica (57).

Quando se utilizam parâmetros laboratoriais, entre os quais a medida da agregabilidade plaquetária, cerca de $20 \%$ dos pacientes com DM apresentam resistência à aspirina $(59,60)$.

Entretanto, estudos adicionais são necessários para estabelecer se a presença de DM é um fator independente para falência terapêutica do uso da aspirina e avaliar a eficácia de doses mais elevadas do agente antiplaquetário na prevenção de eventos cardiovasculares, bem como o uso de agentes alternativos, como o clopidogrel $(36,37)$.

\section{COMENTÁRIOS FINAIS}

$\mathrm{O} \mathrm{DM}$ é um fator de risco equivalente à doença cardiovascular (DCV) e sua presença aumenta de forma significativa a incidência de doença coronariana. A análise detalhada dos estudos sugere que a aspirina em doses baixas é eficaz na prevenção de eventos cardiovasculares em pacientes de alto risco, embora não haja estudos especificamente em pacientes com DM. Nesses indivíduos, as evidências sugerem que o uso de aspirina parece ser menos eficaz, com uma menor redução de eventos cardiovasculares. A redução da eficácia pode ser explicada, em parte, pelos efeitos estruturais da hiperglicemia nas plaquetas e pelo aumento da incidência de fatores relacionados com a falência terapêutica da aspirina.

A presença de complicações microvasculares, principalmente o aumento da EUA, correlaciona-se fortemente com a presença de aterosclerose e, conseqüentemente, DCV. Portanto, o uso regular de aspirina como estratégia de prevenção primária e secundária de DCV deve ser recomendado de forma enfática nesses pacientes, sendo que, adicionalmente, não aumenta o risco de hemorragia retiniana nem interfere na função renal. Por outro lado, embora o benefício renal com redução da albuminúria venha sendo descrito, permanece ainda a necessidade de comprovação.

Em conclusão, o uso de aspirina (75-162 $\mathrm{mg} /$ dia) é fortemente recomendado como estratégia de prevenção cardiovascular em pacientes com DM, apesar da possibilidade de ocorrência de "resistência", cujos mecanismos deverão ser melhor elucidados. Esse entendimento permitirá a adoção de medidas mais eficazes ou ajuste de doses para proteção cardiovascular neste grupo de pacientes.

\section{REFERÊNCIAS}

1. Wild S, Roglic G, Green A, Sicree R, King H. Global prevalence of diabetes: estimates for the year 2000 and projections for 2030. Diabetes Care 2004;27:1047-53. 
2. Ardern Cl, Katzmarzyk PT, Janssen I, Church TS, Blair SN. Revised Adult Treatment Panel III guidelines and cardiovascular disease mortality in men attending a preventive medical clinic. Circulation 2005;112:1481-8.

3. Eidelman RS, Lamas GA, Hennekens CH. The new National Cholesterol Education Program guidelines: clinical challenges for more widespread therapy of lipids to treat and prevent coronary heart disease. Arch Intern Med 2002;162:2033-8.

4. Hennekens $\mathrm{CH}$. Increasing global burden of cardiovascular disease: current knowledge and future directions for research on risk factors: special report: the 1997 American Heart Association's Lewis Atterbury Conner Lecture. Circulation 1998;97:1095-102.

5. Scheffel RS, Bortolanza D, Weber CS, Costa LA, Canani LH, Santos KG, et al. Prevalência de complicações micro e macrovasculares e seus fatores de risco em pacientes com diabetes melito do tipo $2 \mathrm{em}$ atendimento ambulatorial. Rev Assoc Med Bras 2004; $50: 263-7$.

6. US Renal Data System, USRDS 2005 Annual Data Report: Atlas of End-Stage Renal Disease in the United States, National Institute of Health, National Institute of Diabetes and Digestive and Kidney Diseases. Bethesda, MD, 2005.

7. Murussi M, Baglio P, Gross JL, Silveiro SP. Risk factors for microalbuminuria and macroalbuminuria in type 2 diabetic patients. A 9-year follow-up study. Diabetes Care 2002;25(6):1101-3.

8. Bruno RM, Gross JL. Prognostic factors in Brazilian diabetic patients starting dialysis: a 3.6-year follow-up study. J Diabetes Complications 2000;14:226-71.

9. Zaslavsky LM, Pinotti AF, Gross JL. Diastolic dysfunction and mortality in diabetic patients on hemodialysis: a 4.25-year controlled prospective study. J Diabetes Complications 2005; 19:194-200.

10. Valmadrid CT, Klein R, Moss SE, Klein BE. The risk of cardiovascular disease mortality associated with microalbuminuria and gross proteinuria in persons with older-onset diabetes mellitus. Arch Intern Med 2000;160:1093-100.

11. Fioretto $P$, Stehouwer CDA, Chiesura-Corona M, Brocco E, Carraro A, Bortoloso $E$, et al. Heterogeneous nature of microalbuminuria in NIDDM: studies of endothelial function and renal structure. Diabetologia 1998;41:233-6.

12. Stehouwer CDA, Gall MA, Twisk JWR, Knudsen E, Emeis JJ, Parving $\mathrm{HH}$. Increased urinary albumin excretion, endothelial dysfunction, and chronic low-grade inflammation in type 2 diabetes. Diabetes 2002;51:1157-65.

13. Festa A, D'Agostino Jr R, Howard G, Mykkänen L, Tracy RP, Haffner SM. Inflammation and microalbuminuria in non diabetic and type 2 diabetic subjects: The Insulin Resistance Atherosclerosis Study. Kidney Int 2000;58:1703-10.

14. Ceriello A. New insights on oxidative stress and diabetic complications may lead to a causal antioxidant therapy. Diabetes Care 2003;26:1589-96.

15. American Diabetes Association: Standards of medical care in diabetes (Position Statement). Diabetes Care 2005;28:S4-36.

16. Rolka DB, Fagot-Campagna A, Narayan KMV. Aspirin use among adults with diabetes: estimates from the Third National Health and Nutrition Examination Survey. Diabetes Care $2001 ; 24: 197-201$.

17. Sandler DP, Burr FR, Weinberg CR. Nonsteroidal anti-inflammatory drugs and the risk for chronic renal disease. Ann Intern Med 1991; 115:165-72.

18. Funk CD, Funk LB, Kennedy ME, Pong AS, FitzGerald GA. Human platelet/erythroleukemia cell prostaglandin $\mathrm{G} / \mathrm{H}$ synthase: cDNA cloning, expression, and gene chromosomal assignment. FASEB J 1991;15:2304-12.

19. FitzGerald GA. Mechanisms of platelet activation: thromboxane $\mathrm{A} 2$ as an amplifying signal for the others agonists. Am J Cardiol 1991;68:11B-5.

20. Warner TD, Mitchell JA. Cyclooxygenases: new forms, new inhibitors, and lessons from the clinic. FASEB J 2004; 18:790-804.

21. Catella-Lawson F, Reilly MP, Kapoor SC, Cucchiara AJ, DeMarco $\mathrm{S}$, Tournier B, et al. Cyclooxygenase inhibitors and the antiplatelet effects of aspirin. N Engl J Med 2001;345:1809-17.
22. Patrono $C$, Rodríguez LAG, Landolfi R, Baigent C. Low-dose aspirin for the prevention of atherothrombosis. N Engl J Med 2005;353:2373-83.

23. Wu R, Lamontagne $D$, de Champlain J. Antioxidative properties of acetylsalicylic acid on vascular tissues from normotensive and hypertensive rats. Circulation 2002; 105:387-92.

24. Muller DN, Heyssmeyer V, Dechend R, Hampich F, Park JK Fiebeler $A$, et al. Aspirin inhibits NF- $\downarrow B$ and protects from angiotensin II-induce organ damage. FASEB J 2001; 15:1822-4.

25. US Preventive Services Task Force: Aspirin for the primary prevention of cardiovascular events: recommendations and rationale. Ann Intern Med 2002;136:157-60.

26. The Steering Committee of the Physicians' Health Study Research Group: Final report on the aspirin component of the ongoing Physicians' Health Study. N Engl J Med 1989;321:129-35.

27. Peto R, Gray R, Collins R, Wheatley K, Hennekens C, Jamrozik $\mathrm{K}$, et al. Randomized trial of prophylatic daily aspirin in British male doctors. BMJ 1988;296:313-6.

28. The Medical Research Council's General Practice Research Framework. Thrombosis Prevention Trial: randomized trial of low-intensity oral anticoagulation with warfarin and low-dose aspirin in the primary prevention of ischaemic heart disease in men at increased risk. Lancet 1998;351:233-41.

29. Hanson L, Zanchetti A, Carruthers SG, Dahlof B, Elmfelal D, Julius S, et al.; for the HOT study group. Effects of intensive blood-pressure lowering and low-dose aspirin in patients with hypertension: principal results of the Hypertension Optimal Treatment (HOT) randomized trial. Lancet 1998;351:1755-62.

30. Collaborative Group of the Primary Prevention Project. Lowdose aspirin and vitamin $E$ in people at cardiovascular risk: a randomized trial in general practice. Lancet 2001;357:89-95.

31. Sacco M, Pellegrini F, Roncaglioni MC, Avanzini F, Tognoni G, Nicolucci A; on behalf of the PPP Collaborative Group. Primary prevention of cardiovascular events with low-dose aspirin and vitamin E in type 2 diabetic patients. Results of the Primary Prevention Project (PPP) trial. Diabetes Care 2003;26:3264-72

32. Eidelman RS, Herbert PR, Weisman SM, Hennekens CH. An update on aspirin in the primary prevention of cardiovascular disease. Arch Intern Med 2003;163:2006-10.

33. Ridker PM, Cook NR, Lee IM, Gordon D, Gaziano JM, Manson $\mathrm{JE}$, et al. A randomized trial of low-dose aspirin in the primary prevention of cardiovascular disease in women. N Engl J Med 2005;352:1366-8.

34. Berger JS, Roncaglioni MC, Avanzini F, Pangrazzi I, Tognoni $\mathrm{G}$, Brown DL. Aspirin for the primary prevention of cardiovascular events in women and men. A sex-specific metaanalysis of randomized controlled trials. JAMA 2006;295:306-13.

35. Antithrombotic Trialists' Collaboration: Collaborative metaanalysis of randomized trials of antiplatelet therapy for prevention of death, myocardial infarction, and stroke in highrisk patients. BMJ 2002;324:71-86.

36. Bhatt DL, Marso SP, Hirsch AT, Ringleb PA, Hacke W, Topol EJ. Amplified benefit of clopidogrel versus aspirin in patients with diabetes mellitus. Am J Cardiol 2002;90:625-8.

37. Bhatt DL, Fox KA, Hacke W, Berger PB, Black HR, Boden WE, et al.; CHARISMA Investigators. Clopidogrel and aspirin versus aspirin alone for the prevention of atherothrombotic events. N Engl J Med 2006;354:1706-17.

38. Donadio JV, Ilstrup DM, Holley KE, Romero JC. Plateletinhibitor treatment of diabetic nephropathy: a 10-year prospective study. Mayo Clinic Proc 1988;63:3-15.

39. Hopper $A H$, Tindall H, Davies A. Administration of aspirindipyridamole reduces proteinuria in diabetic nephropathy. Nephrol Dial Transplant 1989;4:140-3.

40. Khajehdehi $P$, Roozbeh J, Mostafavi H. A comparative randomized and placebo-controlled short-term trial of aspirin an dypiridamole for overt type-2 diabetic nephropathy. Scand J Urol Nephrol 2002;36:145-8. 
41. Hansen HP, Gaede PH, Jensen BR, Parving HH. Lack of impact of low-dose acetylsalicylic acid on kidney function in type 1 diabetic patients with microalbuminuria. Diabetes Care 2000;23:1742-5.

42. Gaede $\mathrm{P}$, Hansen HP, Parving $\mathrm{HH}$, Pedersen O. Impact of lowdose acetylsalicylic acid on kidney function in type 2 diabetic patients with elevated urinary albumin excretion rate. Nephrol Dial Transplant 2003;18:539-42.

43. Camargo EG, Weinert L, Lavinsky J, Gross JL, Silveiro SP Efeito da aspirina em baixa dosagem sobre a excreção urinária de albumina e a taxa de filtração glomerular em pacientes com diabete melito tipo 2 e microalbuminúria. Arq Bras Endocrinol Metab 2005;49(supl. 2):S874.

44. Pitt B, Yusuf S; for the SOLVD Investigators. Studies of left ventricular dysfunction (SOLVD): subgroup results. J Am Coll Cardiol 1992;19:215A.

45. Peterson JG, Topol E, Sapp SK, Young JB, Lincoff AM, Lauer MS. Evaluation of the effects of aspirin combined with angiotensin-converting enzyme inhibitors in patients with coronary artery disease. Am J Med 2000;109:371-7.

46. Teo KK, Yusuf S, Pfeffer M, Kober L, Hall A, Pogue J, et al.; for the ACE Inhibitors Collaborative Group. Effects of long-term treatment with angiotensin-converting-enzyme inhibitors in the presence or absence of aspirin: a systematic review. Lancet 2002;360:1037-43.

47. Fossum E, Moan A, Kjeldsen SE, Devereux RB, Julius S, Snapinn SM, et al.; for the Losartan Intervention for Endpoint Reduction in Hypertension (LIFE) Study. The effect of losartan versus atenolol on cardiovascular morbidity and mortality in patients with hypertension taking aspirin. J Am Coll Cardiol 2005;46:770-5.

48. Early Treatment Diabetic Retinopathy Study (ETDRS) Research Group. Effects of aspirin treatment on diabetic retinopathy. ETDRS report number 8. Ophthalmology 1991;98:757-65.

49. Cusick M, Meleth AD, Agron E, Fisher MR, Reed GF Knafterud GL, et al.; for the Early Treatment Diabetic Retinopathy Study (ETDRS) Research Group. Associations of mortality and diabetes complications in patients with type 1 and type 2 diabetes. ETDRS report number 27. Diabetes Care 2005;28:617-25.

50. Sanderson S, Emery J, Baglin T, Kinmonth AL. Aspirin resistance and its clinical implications. Ann Intern Med 2005; $142: 370-80$

51. Hankey GJ, Eikelboom JW. Aspirin resistance. Lancet 2006;367:606-17.
52. Schwartz KA, Schwartz DE, Ghosheh K, Reeves MJ, Barber K, Defranco A. Compliance as a critical consideration in patients who appear to be resistant to aspirin after healing of myocardial infarction. Am J Cardiol 2005;95:973-5.

53. Gum PA, Kottke-Marchant K, Poggio ED, Gurm H, Welsh PA, Brooks $\mathrm{L}$, et al. Profile and prevalence of aspirin resistance in patients with cardiovascular disease. Am J Cardiol 2001;88:230-5.

54. Friend M, Vucenik I, Miller M. Research pointers: platelet responsiveness to aspirin in patients with hyperlipidemia. BMJ 2003;326:82-3.

55. Bornstein NM, Karepov VG, Aronovich BD, Gorbulev AY, Treves TA, Korczyn A. Failure of aspirin treatment after stroke. Stroke 1994;25:275-7.

56. Watala C, Golanski J, Pluta J, Boncler M, Rozalski M, Luzak B, et al. Reduced sensitivity of platelets from type 2 diabetic patients to acetylsalicylic acid (aspirin)- its relation to metabolic control. Thromb Res 2004;113:101-13.

57. Watala C, Pluta J, Golanski J, Rozalski M, Czyz M, Trojanowski $Z$, et al. Increased protein glycation in diabetes mellitus is associated with decreased aspirin-mediated protein acetylation and reduced sensitivity of blood platelets to aspirin. $\mathbf{J}$ Mol Med 2005;83:148-58.

58. Colwell JA, Nesto RW. The platelet in diabetes. Diabetes Care 2003;26:2181-8.

59. Fateh-Moghadam S, Plöckinger U, Cabeza N, Htun P, Reuter $\mathrm{T}$, Ersel $\mathrm{S}$, et al. Prevalence of aspirin resistance in patients with type 2 diabetes. Acta Diabetol 2005;42:99-103.

60. Mehta SS, Silver RJ, Aaronson A, Abrahamson M, Goldfine AB. Comparison of aspirin resistance in type 1 versus type 2 diabetes mellitus. Am J Cardiol 2006;97:567-70.

\section{Endereço para correspondência:}

Sandra Pinho Silveiro

Serviço de Endocrinologia

Hospital de Clínicas de Porto Alegre

Rua Ramiro Barcelos 2350, prédio 12, 4\% andar

90035-003 Porto Alegre, RS

E-mail: sandrasilveiro@terra.com.br 